\title{
Psychological Aspects Among Children and Adolescents of Orphanages at Assiut City
}

\author{
Bakheeta Abd El-Aziz Mohammed $^{1}$, Yasser Mohamed Badereldin ${ }^{2}$ \& Amera Ezzat Abd El-Naser ${ }^{3}$
}

1. Demonstrator of Psychiatric and Mental Health Nursing, Faculty of nursing, Assiut University.

2. Professor of Psychiatry Medicine, Faculty of Medicine, Assiut University.

3. Lecturer of Psychiatric and Mental Health Nursing, Faculty of nursing, Assiut University.

\begin{abstract}
It is a catastrophe for any child the death of one or both parents. Children may feel with depression, hopelessness, loneliness, anger, confusion, helplessness, anxiety and a fear of being alone. This study aimed to assess psychological aspects among children and adolescents of orphanages at Assiut City. Subjects and method: A descriptive research design used to conduct this study. A purposive sample was used. The present study included 120 subjects. Setting: The study was conducted at Lillian Trasher, Dar El Hanan, and Dar El Safa. Tools of study included demographic data, multidimensional child and adolescent depression scale (MCADS) and child behavior checklist (parent form). Results of the study: Three fifth of studied orphans $(60 \%)$ in the age group from ( $>12-$ 17 years old).Also $65 \%$ of studied orphans were males., there were statistically significant relations between age and most of depressive symptoms; also the most predominant problems were aggression, anxiety/depression and attention problem whereas thought problem was the least and internalizing behavioral problems were higher than externalizing behavioral problems (37.5\% versus $34.2 \%)$. Conclusion: most orphans had different psychological problems and the prevalence of psychological problems/symptoms was more in adolescence age than child ages.Recommendation: Psychosocial counseling program can be designed and administered to orphan children and adolescents to improve depressive symptoms and their psychological status.
\end{abstract}

\section{Key words: Psychological aspects, Orphan, Children, Adolescents \& Orphanages.}

\section{Introduction}

Children are fundamental resources and the future of a nation. The society should offer them sufficient opportunities for their proper physical, mental, social and moral growth as well as personality development and education (Akram et al., 2015).

Orphan hood can be a stressful experience and can adversely affect the well-being of children (Yendork \& Somhlaba, 2015). The orphan child is whom one or both parents have been disappearing, desertion, or unable to provide care (Berns \& Nelson, 2015 ).

Worldwide, an estimated 153 million children, ranging from newborns to those aged 17 years, have lost one or both of their parents, and every day 5760 more children lose at least one parent (Mohammadzadeh et al, 2017).

Also there are 1.7 million orphans in Egypt, and the most of Egyptian Foster care sector is anongovernmental relying on private support with minimal government supervision (Mohammed et al., 2013).

The most intensely painful experiences any human being can suffer are loss of a loved person (Coghlan, 2014). When losing a parent occurs in childhood or adolescence, a child may fail in mourning sufficiently and later in life may often appear with symptoms of depression or inability to form close relationships during puberty (Shaik Omar, 2016).

The experience of family loss and the lack of social support available to orphans can lead to anxiety, poor self-esteem, depression and delinquent behavior (Jameel et al., 2015).

Moreover they later find it difficult to relate to the outside world, struggle to communicate and express their emotions, struggle to deal with the past and are apprehensive towards the future (Kostopoulos, 2014).

A huge number of orphans universally are alive with no natural relations due to diversity of causes together with: death of parents, separation between parents, domestic injurious treatment and disregard. The most frequent causes of separation of children from parental care include poverty, lack of access to basic services, abuse, neglect, disease, disabilities, and emergencies (Blaikie et al., 2014).

If any child does not have such relatives who can support him, then the orphanages are only one way to provide him/her shelter, health care, food, education and accommodation (Akram et al., 2015).

Depression is a common reaction in children who are separated from their parents (Hopkins, 2008). In this respect, it was reported that, depression in children may involve fewer symptoms in total than depression in older age groups. Adolescents report more sleep and appetite disturbances, and suicidal ideation than younger children. However, adolescents still exhibit more behavioral problems than adults (Birmaher\& Brent, 2010).

Also stress of separation may be shown in symptoms of confusion, anxiety, depression, and behavioral 
disorders such as disobedience. The same symptoms may cause learning problems. Children who are frustrated, fearful, and depressed may fail to concentrate in class and therefore perform badly. Failure by the school and the home systems to recognize these symptoms and address them will aggravate the child's psychological problems (Cluver et al., 2007).

Many orphans and vulnerable children (OVC) internalize problems, resulting in symptoms of depression, anger, hopelessness, loneliness, low selfesteem, and suicide ideation (Boris et al., 2008). Internalizing problems often called emotional problems indicate internal manifestation of psychological distress (e.g. depression and anxiety) rather than acting them out in the environment (e.g. behavioral problems, conduct problems) (Boadu , 2015). They also externalize psychosocial distress through acts of delinquency, increased sexual activity, and more passive displays such as social withdrawal and difficulties concentrating in school, such behaviors will place an entire generation of OVC at increased risk for further harm. (De Witt\& Lessing, 2010).

\section{Significance of the study}

Most studies have focused on physical aspects of orphans either needs or problems. Despite the serious potential consequences of bereavement in childhood, including risk for depression, maladaptive grief, and other emotional and behavioral problems (Melhem et al., 2011).

There are minimal studies on the psychological health of children, orphans in general and those brought up in institutions in particular suffer from many social, psychological and economic problems (Boris, 2012). These problems continue into the future, causing various disabilities during adulthood and cause great harm to people and even society (Mohammadzadeh et al, 2017).So, the present study aimed to assess psychological aspects among orphan children and adolescents of orphanages and this study may help the nursing and medical researchers to develop knowledge regarding psychological aspects among orphan children.

\section{Aim of the study \\ This present study aimed to \\ Assess psychological aspects among children and adolescents of orphanages at Assiut City. \\ Research questions \\ The present study seek out to answer the following questions \\ - Could orphan hood affects the psychological aspects of orphan children and adolescents?}

- Could orphan hood affects the behavior of orphan children and adolescents?

\section{Subjects \& Method}

Research design

A descriptive research design was used in this study. Study settings

The study was conducted in three orphanages and they consider the major at Assiut city and these orphanages serve Assiut Governorate:

- Lillian Trasher Orphanage Center (males and females) in El-Fath SharQ El-Khazan. It is a big institution containing a lot of facilities and buildings: Administrative building, living building one for males and one for females and each building containing T.V room, toilet in each floor, internal kitchen, hospital, church, primary school learning children who are residents in the orphanage and other children from neighboring areas, main kitchen, carpentry workshop, farm, bakery, Playground and swimming pool in addition, wide spaces between buildings.

- Dar El Hanan(males) in El-Fath SharQ ElKhazan.It is consisted from two large sectors, sleeping rooms, two rooms for specialists, iron room, laundry room, dressmaking room, carpentry workshop, small playground and small area for prayer as a mosque.

- Dar El Safa(females) in Thabet Street at Assiut City. It is old building from Thabet Pasha days 1939.It is containing four sleeping rooms, T.V room, Toilets, Hall, Internal and external kitchen, Library.

\section{Subjects}

1-All orphaned children and adolescents from 7-17 years (school age), who were residents in the previous three mentioned homes were included $(\mathrm{n}=120) 78$ males and 42 females according to selection criteria.

Orphaned children were divided into children (7-12 years) and adolescents (more than 12-17 years) in line with theories of human development that refer to the different stages of development of these groups

(Berk 2002, Louw \& Louw 2007).

Criteria of selection

Inclusion criteria

-Age from 7-17 years old.

-Agree to participate in the study.

Exclusion criteria

- Orphan children and adolescents who have any physical or mental disabilities.

Data collection tools: The study tools consisted of three parts

Part (1): Demographic data: Developed by the researcher, It was included (name, age, sex, place, educational level). 
Part (2): The Multidimensional Child and Adolescent Depression Scale (MCADS): This scale was developed by Abdel-Khalek (2003): it has two compatible Arabic and English versions. The Arabic version was used and it consists of 40 items

\section{Which divided into eight dimensions}

Pessimism, weak concentration, sleep problems, anhedonia, fatigue, loneliness, low self- esteem and somatic complaints.

Each dimension is assessed by five items: so MCADS has 40 brief statements answered on a three point intensity scale i.e., "none"=1,"sometimes"=2,"a lot" $=3$. which mostly describes the emotional state of the child. Except for statements 1,6,11,16,23,34,\&35 which were scored as follows : none $=3$, sometimes $=2$ $\&$ a $\operatorname{lot}=1$.

With regard to the reliability of the scale, Cronbach's alpha ranged from 0.63 to 0.92 , while the test retest reliability ranged between 0.56 and 0.87 . The criterion- related validity ranged between 0.30 and 0.85 . Mean \pm SD for every subscales and total scores computed .The higher score the more problem.

Part (3): Child behavior checklist, parent form (developed by Achenbach \& Rescorla, 2001): This scale was for the children ages from (6-18) year and consists of 113 questions. Arabic version was used, it was translated by (Mohammed, 2001 \& Abdel Khalek, 2006).It has two main components: I-Total competence scale: This computed as a sum of activities level, social relationship, and school performance (competence subscale).The scoring system of the scale was based on T-score. If T-score is more than 40 , it will consider in the normal range, while 37-40 represents borderline score and T-score that is less than 37 which represents the abnormal level.

II-The problem scales: These subscales compromised of three broad constructs they are internalizing behavioral problems, externalizing behavioral problems and a total problem scales score.

- The internalizing behavioral problems consist of the sum of the scores of 3 subscales that include withdrawn, somatic complaints and anxiety and depression subscales.

- Externalizing behavioral problems consist of the sum of the scores of 2 subscales that include delinquent and aggressive behavior subscales.

- The social problems, thought problems, and attention problems scales are not included in either the internalizing or externalizing groupings, but were included in the total problems score, which include all core syndromes as demonstrated in the table.

The participants responded into (3) point likert scale (never $=$ zero, sometimes $=1$, and always $=2$ ). For total problems, externalizing behavioral problems and internalizing behavioral problems, if $\mathrm{T}$-scores less than 60 it will consider in the normal range, 60-63 score represents borderline score, and scores which are greater than 63 will be in the abnormal level.

Child behavior checklist (Parent Form)

\begin{tabular}{|c|c|c|}
\hline Subscales & $\begin{array}{l}\text { No of } \\
\text { items }\end{array}$ & No. of questions \\
\hline \multicolumn{3}{|c|}{ I. I. Total competence scales } \\
\hline $\begin{array}{l}\text { 1-Activities level } \\
\text { subscale }\end{array}$ & 8 & $\begin{array}{c}(\mathrm{I}=\mathrm{A}, \mathrm{B}, \mathrm{C}),(\mathrm{II}=\mathrm{A}, \mathrm{B}, \mathrm{C}) \text { and } \\
(\mathrm{IV}=\mathrm{A}, \mathrm{B})\end{array}$ \\
\hline $\begin{array}{l}\text { 2-Social } \\
\text { relationship } \\
\text { subscale }\end{array}$ & 6 & $\begin{array}{c}(\mathrm{III}=\mathrm{A}, \mathrm{B}),(\mathrm{V}=1,2) \text { and } \\
(\mathrm{VI}=\mathrm{A}, \mathrm{B})\end{array}$ \\
\hline $\begin{array}{l}\text { 3-School } \\
\text { performance } \\
\text { subscale }\end{array}$ & 4 & $(\mathrm{VII}=1,2,3,4)$ \\
\hline \multicolumn{3}{|l|}{ II. Problem scales } \\
\hline \multicolumn{3}{|c|}{ A)Internalizing behavioral problems } \\
\hline 1.Withdrawn & 9 & $\begin{array}{l}(42,65,69,75,80,88,102,103,111 \\
)\end{array}$ \\
\hline 2.somatic complain & 9 & $\begin{array}{l}(51,54,56 \mathrm{a}, 56 \mathrm{~b}, 56 \mathrm{c}, 56 \mathrm{~d}, 56 \mathrm{e}, 56 \mathrm{f} \\
, 56 \mathrm{~g})\end{array}$ \\
\hline $\begin{array}{l}\text { 3.Anxiety and } \\
\text { Depression }\end{array}$ & 14 & $\begin{array}{l}(12,14,31,32,33,34,35,45,50,52, \\
71,89,103,112)\end{array}$ \\
\hline \multicolumn{3}{|c|}{ B)Externalizing behavioral problems } \\
\hline $\begin{array}{l}\text { 4.Delinquent } \\
\text { behavior }\end{array}$ & 13 & $\begin{array}{l}(26.39,43,63,67,72,81,82,90,96, \\
101,105,106)\end{array}$ \\
\hline $\begin{array}{l}\text { 5.Aggressive } \\
\text { behavior }\end{array}$ & 20 & $\begin{array}{l}(3,7,16,19,20,21,22,23,, 27,37,5 \\
7,68,74,86,87,93,94,95,97,104) \\
\end{array}$ \\
\hline \multicolumn{3}{|c|}{ C)Total problem scale } \\
\hline 6.Thought problem & 7 & $(9,40,66,70,80,84,85)$ \\
\hline 7.Social problem & 8 & $(1,11,25,38,48,55,62,64)$ \\
\hline 8.Attention problem & 11 & $\begin{array}{c}(1,8,10,13,17,41,45,46,61,62, \\
, 80)\end{array}$ \\
\hline In addition to Int & ing & externalizing subscales \\
\hline
\end{tabular}

Steps of the study (Method)

Ethical consideration

- After appraisal of the protocol of the study by the Ethical and Scientific Committee, an official letter was issued from the Dean Faculty of Nursing; Assiut University to the Agent of Ministry Social Solidarity Directorate. Then letter was sent to mangers of the previous mentioned orphanage homes to get their permission for data collection and conducting the research.

- The aim of the study was explained to the participants before starting data collection.

- Issues of confidentiality, anonymity, and data security were maintained during the research.

- Informed oral consent was taken from all participants for their approval to share in this study.

- Risk-benefit assessment: There is no risk during application of the research

Field work

Operational phase: The actual data collection was conducted for six months from January 2017 till end of June 2017 after getting permission from the previous mentioned orphanage homes mangers that follow Assiut City. 


\section{Implementation phase}

- A pilot study was conducted on 18 orphan children and adolescents. The aim of the pilot study was to detect any particular problem in the items clarity, feasibility and applicability of the tools. No change was made in the assessment tools, so the selected sample of the pilot study was included in the main study.

- The researcher interviewed each participant individually after taken oral consent and started with ice breaking open friendly discussion to gain his/her trust.

- Then the researcher explained the aim of the study to subjects asked him/ her questions in a simple Arabic language.

- Orphan Children and Adolescents were interviewed two days per week.
- Each interview took about 25-35 minutes to fill the Multidimensional Child and Adolescent Depression Scale for each one.

- Half an hour to fill Child behavior checklist, Parent Form from caregivers.

- The researcher assured them that their names will not be written.

\section{Statistical analysis}

The data were tabulated and statistically analyzed using SPSS software (Statistical Package for the Social Sciences, version 19). For quantitative data, the frequencies, percentages, mean and standard deviation were calculated. For qualitative data, comparison between two groups and more was done using Chi-square test $(\chi 2)$. Kruskal Wallis test and Mann-Whitney test. P- Value is considered significant if it was less than 0.05 .

Results: The main results yielded by this study were:

Table (1): Demographic data of the studied orphan in Assiut City (N=120).

\begin{tabular}{|c|c|c|}
\hline & No. $(n=120)$ & $\%$ \\
\hline \multicolumn{3}{|l|}{ Age: (years) } \\
\hline $7-12$ years & 48 & 40.0 \\
\hline$>12-17$ years & 72 & 60.0 \\
\hline \multicolumn{3}{|l|}{ Sex } \\
\hline Male & 78 & 65.0 \\
\hline Female & 42 & 35.0 \\
\hline \multicolumn{3}{|l|}{ Level of education } \\
\hline Primary & 49 & 40.8 \\
\hline Preparatory & 43 & 35.8 \\
\hline Secondary & 13 & 10.8 \\
\hline Technical & 15 & 12.5 \\
\hline \multicolumn{3}{|l|}{ Place } \\
\hline Lillian Trasher & 93 & 77.5 \\
\hline Al-Hanan & 15 & 12.5 \\
\hline Al-Safa & 12 & 10.0 \\
\hline
\end{tabular}

Table (2): Descriptive statistics of psychological aspects among the studied orphans in Assiut city ( $N=120)$.

\begin{tabular}{|c|c|}
\hline Variables & Mean \pm SD \\
\hline \multicolumn{2}{|l|}{ *Domains of competence scale } \\
\hline Activities levels & $6.06 \pm 4.06$ \\
\hline Social relationship & $8.65 \pm 2.91$ \\
\hline School performance & $6.47 \pm 3.69$ \\
\hline Total competence scale & $21.18 \pm 7.91$ \\
\hline \multicolumn{2}{|l|}{ *Domains of problem scale } \\
\hline Internal problems: & $12.54 \pm 8.90$ \\
\hline Withdrawn & $4.32 \pm 3.72$ \\
\hline Somatic complaints behavior & $1.57 \pm 1.28$ \\
\hline Anxiety/ depression & $6.66 \pm 5.12$ \\
\hline External problems: & $15.33 \pm 13.32$ \\
\hline Delinquent behavior & $4.58 \pm 4.86$ \\
\hline Aggressive behavior & $10.76 \pm 9.02$ \\
\hline
\end{tabular}




\begin{tabular}{|l|c|}
\hline \multicolumn{1}{|c|}{ Variables } & Mean \pm SD \\
\hline Social problems & $3.86 \pm 3.24$ \\
\hline Thought problems & $2.58 \pm 3.42$ \\
\hline Attention problems & $5.75 \pm 4.69$ \\
\hline Total problem scale & $40.07 \pm 30.87$ \\
\hline
\end{tabular}

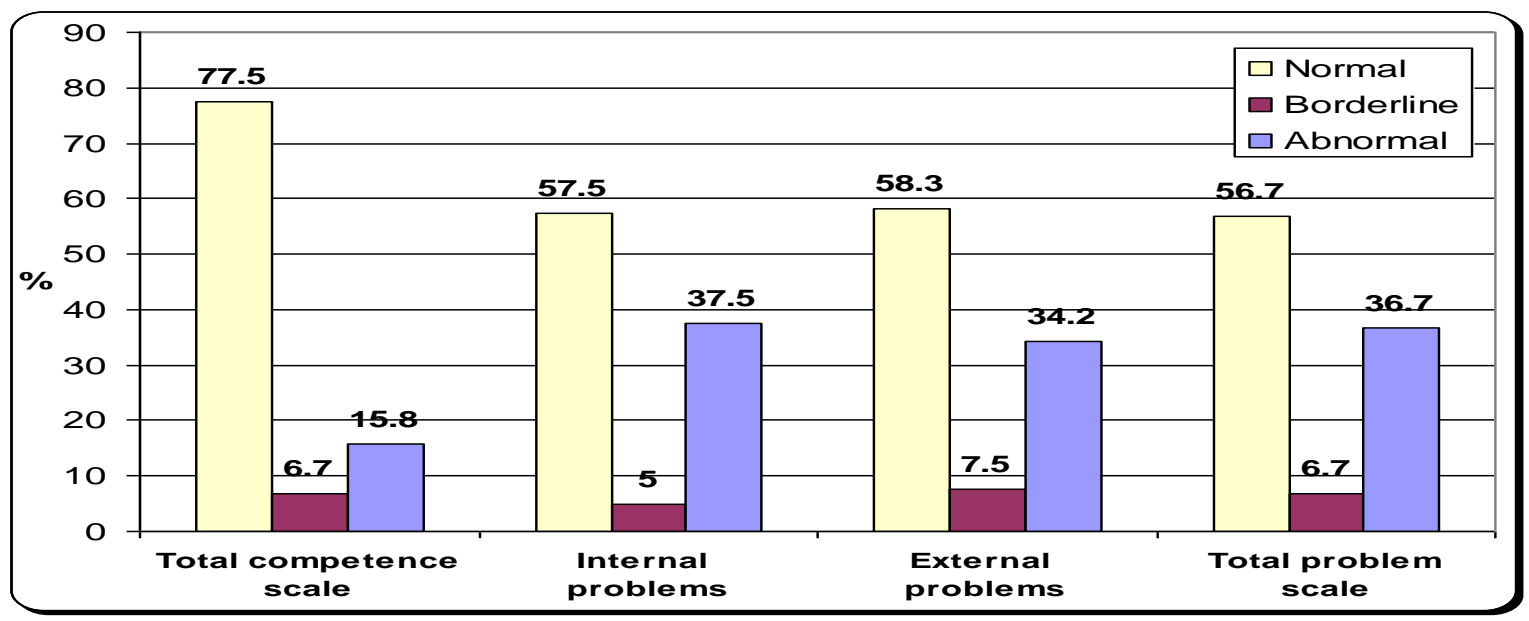

Figure (1): Distribution of the studied orphans regarding Child behavior in Assiut City ( $N=120)$.

Table (3): Relation between demographic data and domains of total competence scale among the studied orphans in Assiut City ( $\mathrm{N}=120)$.

\begin{tabular}{|c|c|c|c|c|}
\hline \multirow[t]{2}{*}{ Variables } & Activities level & $\begin{array}{c}\text { Social } \\
\text { relationship }\end{array}$ & $\begin{array}{c}\text { School } \\
\text { performance }\end{array}$ & $\begin{array}{c}\text { Total competence } \\
\text { scale }\end{array}$ \\
\hline & Mean \pm SD & Mean \pm SD & Mean \pm SD & Mean \pm SD \\
\hline \multicolumn{5}{|l|}{ Age: (years) } \\
\hline $7-12$ years & $4.73 \pm 3.47$ & $8.56 \pm 2.58$ & $6.81 \pm 3.22$ & $20.10 \pm 6.46$ \\
\hline$>12-17$ years & $6.94 \pm 4.21$ & $8.71 \pm 3.13$ & $6.24 \pm 3.98$ & $21.89 \pm 8.72$ \\
\hline P-value & 0.001* & 0.766 & 0.271 & 0.548 \\
\hline \multicolumn{5}{|l|}{ Sex: } \\
\hline Male & $5.97 \pm 3.32$ & $8.03 \pm 2.37$ & $5.94 \pm 3.27$ & $19.94 \pm 6.14$ \\
\hline Female & $6.21 \pm 5.21$ & $9.81 \pm 3.45$ & $7.45 \pm 4.23$ & $23.48 \pm 10.13$ \\
\hline P-value & 0.907 & 0.001* & 0.040* & 0.039* \\
\hline \multicolumn{5}{|l|}{ Place } \\
\hline Lillian Trasher & $6.18 \pm 3.70$ & $8.99 \pm 2.83$ & $7.15 \pm 3.48$ & $22.32 \pm 7.91$ \\
\hline Al-Hanan & $6.87 \pm 4.61$ & $7.93 \pm 2.58$ & $3.27 \pm 2.34$ & $18.07 \pm 6.04$ \\
\hline Al-Safa & $4.08 \pm 5.65$ & $6.92 \pm 3.40$ & $5.17 \pm 4.41$ & $16.17 \pm 7.44$ \\
\hline P-value & 0.110 & 0.073 & 0.000* & 0.014* \\
\hline \multicolumn{5}{|c|}{ Level of education: } \\
\hline Primary & $5.02 \pm 3.44$ & $8.65 \pm 2.57$ & $6.98 \pm 3.19$ & $20.65 \pm 6.31$ \\
\hline Preparatory & $7.40 \pm 4.57$ & $8.63 \pm 3.46$ & $5.86 \pm 3.75$ & $21.88 \pm 9.79$ \\
\hline Secondary & $5.85 \pm 2.27$ & $9.23 \pm 2.86$ & $9.85 \pm 4.02$ & $24.92 \pm 6.95$ \\
\hline Technical & $5.80 \pm 4.83$ & $8.20 \pm 2.46$ & $3.60 \pm 1.92$ & $17.60 \pm 6.14$ \\
\hline P-value & 0.030* & 0.608 & 0.000* & $0.042 *$ \\
\hline
\end{tabular}

Mann-Whitney test (for age and sex)

Kruskal Wallis test (for place and level of education)

* Statistical significant difference $(P<0.05)$

N.B: The lower score the more problems 
Table (4): Relation between demographic data and domains of total problems scale among the studied orphans in Assiut City ( $\mathbf{N = 1 2 0})$.

\begin{tabular}{|c|c|c|c|c|c|c|c|c|c|c|c|}
\hline \multirow[t]{2}{*}{ Variables } & $\begin{array}{c}\text { Internal } \\
\text { Problems }\end{array}$ & Withdrawn & \begin{tabular}{|c|} 
Somatic \\
complaints \\
behavior
\end{tabular} & $\begin{array}{c}\text { Anxiety/ } \\
\text { Depression }\end{array}$ & $\begin{array}{c}\text { External } \\
\text { Problems }\end{array}$ & $\begin{array}{c}\text { Delinquent } \\
\text { Behavior }\end{array}$ & $\begin{array}{c}\text { Aggressive } \\
\text { Behavior }\end{array}$ & $\begin{array}{c}\text { Social } \\
\text { problems }\end{array}$ & $\begin{array}{l}\text { Thought } \\
\text { Problems }\end{array}$ & $\begin{array}{l}\text { Attention } \\
\text { problems }\end{array}$ & \begin{tabular}{|c|} 
Total \\
problem \\
score \\
\end{tabular} \\
\hline & $\begin{array}{c}\text { Mean } \pm \\
\text { SD }\end{array}$ & $\begin{array}{c}\text { Mean } \pm \\
\text { SD }\end{array}$ & $\begin{array}{c}\text { Mean } \pm \\
\text { SD }\end{array}$ & $\begin{array}{c}\text { Mean } \pm \\
\text { SD }\end{array}$ & $\begin{array}{c}\text { Mean } \pm \\
\text { SD }\end{array}$ & $\begin{array}{c}\text { Mean } \pm \\
\text { SD }\end{array}$ & $\begin{array}{c}\text { Mean } \pm \\
\text { SD }\end{array}$ & $\begin{array}{c}\text { Mean } \pm \\
\text { SD }\end{array}$ & $\begin{array}{c}\text { Mean } \pm \\
\text { SD }\end{array}$ & $\begin{array}{c}\text { Mean } \pm \\
\text { SD }\end{array}$ & $\begin{array}{c}\text { Mean } \pm \\
\text { SD }\end{array}$ \\
\hline \multicolumn{12}{|l|}{ Age: (years) } \\
\hline 7 - 12 years & $\begin{array}{c}11.77 \pm \\
8.31\end{array}$ & $3.73 \pm 3.31$ & $1.50 \pm 1.09$ & $6.54 \pm 5.21$ & $\begin{array}{c}13.65 \pm \\
13.15 \\
\end{array}$ & $3.25 \pm 3.94$ & $\begin{array}{c}10.40 \pm \\
9.53\end{array}$ & $\begin{array}{c}.08 \pm \\
3.49 \\
\end{array}$ & $\begin{array}{c}2.98 \pm \\
3.47 \\
\end{array}$ & $\begin{array}{c}5.48 \pm \\
4.66 \\
\end{array}$ & $\begin{array}{c}37.96 \pm \\
30.26\end{array}$ \\
\hline$>12-17$ years & $\begin{array}{c}13.06 \pm \\
9.30\end{array}$ & $4.71 \pm 3.94$ & $1.61 \pm 1.40$ & $6.74 \pm 5.09$ & $\begin{array}{c}16.46 \pm \\
13.41\end{array}$ & $5.46 \pm 5.22$ & $\begin{array}{c}11.00 \pm \\
8.73\end{array}$ & $\begin{array}{c}3.71 \pm \\
3.07\end{array}$ & $\begin{array}{c}2.32 \pm \\
3.38\end{array}$ & $\begin{array}{c}5.93 \pm \\
4.74\end{array}$ & $\begin{array}{c}41.47 \pm \\
31.39 \\
\end{array}$ \\
\hline P-value & 0.521 & 0.198 & 0.864 & 0.743 & 0.148 & 0.008* & 0.482 & 0.867 & 0.284 & 0.544 & 0.361 \\
\hline \multicolumn{12}{|l|}{ Sex: } \\
\hline Male & $\begin{array}{c}15.36 \pm \\
9.38\end{array}$ & $5.47 \pm 3.83$ & $1.42 \pm 1.44$ & $8.46 \pm 5.22$ & $\begin{array}{c}20.12 \pm \\
13.57 \\
\end{array}$ & $6.26 \pm 5.11$ & $\begin{array}{c}13.86 \pm \\
9.22\end{array}$ & $\begin{array}{c}4.76 \pm \\
3.45 \\
\end{array}$ & $\begin{array}{c}3.67 \pm \\
3.75 \\
\end{array}$ & $\begin{array}{c}7.23 \pm \\
4.73 \\
\end{array}$ & $\begin{array}{c}51.13 \pm \\
31.68 \\
\end{array}$ \\
\hline Female & $\begin{array}{c}7.31 \pm \\
4.66\end{array}$ & $2.17 \pm 2.32$ & $1.83 \pm 0.88$ & $3.31 \pm 2.68$ & $\begin{array}{c}6.45 \pm \\
6.70\end{array}$ & $1.45 \pm 2.01$ & $5.00 \pm 4.93$ & $\begin{array}{c}2.19 \pm \\
1.93\end{array}$ & $\begin{array}{c}0.57 \pm \\
1.09\end{array}$ & $\begin{array}{c}3.00 \pm \\
3.15\end{array}$ & $\begin{array}{c}19.52 \pm \\
14.66\end{array}$ \\
\hline P-value & $0.000 *$ & $0.000 *$ & $0.016 *$ & $0.000 *$ & $0.000 *$ & $0.000 *$ & 0.000* & 0.000* & $0.000 *$ & 0.000* & $0.000 *$ \\
\hline \multicolumn{12}{|l|}{ Place } \\
\hline $\begin{array}{l}\text { Lillian } \\
\text { Trasher }\end{array}$ & $\begin{array}{c}13.26 \pm \\
9.23\end{array}$ & $4.49 \pm 3.89$ & $1.66 \pm 1.29$ & $7.11 \pm 5.36$ & $\begin{array}{c}15.92 \pm \\
13.96\end{array}$ & $4.58 \pm 4.96$ & $\begin{array}{c}11.34 \pm \\
9.53\end{array}$ & $\begin{array}{c}4.17 \pm \\
3.47 \\
\end{array}$ & $\begin{array}{c}2.78 \pm \\
3.71 \\
\end{array}$ & $\begin{array}{c}6.15 \pm \\
4.91 \\
\end{array}$ & \begin{tabular}{|c|}
$42.29 \pm$ \\
32.60 \\
\end{tabular} \\
\hline Al-Hanan & $\begin{array}{c}12.20 \pm \\
8.35 \\
\end{array}$ & $4.27 \pm 3.43$ & $1.60 \pm 1.30$ & $6.33 \pm 4.50$ & $\begin{array}{c}16.00 \pm \\
12.04 \\
\end{array}$ & $6.07 \pm 5.16$ & $9.93 \pm 7.55$ & $\begin{array}{c}2.87 \pm \\
2.26 \\
\end{array}$ & $\begin{array}{c}2.60 \pm \\
2.20 \\
\end{array}$ & $\begin{array}{c}5.27 \pm \\
3.92 \\
\end{array}$ & $\begin{array}{c}38.93 \pm \\
26.61 \\
\end{array}$ \\
\hline Al-Safa & $\begin{array}{c}7.42 \pm \\
4.87 \\
\end{array}$ & $3.00 \pm 2.52$ & $0.83 \pm 1.03$ & $3.58 \pm 2.39$ & $\begin{array}{c}9.92 \pm \\
8.38 \\
\end{array}$ & $2.67 \pm 2.84$ & $7.25 \pm 5.67$ & $\begin{array}{c}2.67 \pm \\
1.61 \\
\end{array}$ & $\begin{array}{c}1.00 \pm \\
1.48 \\
\end{array}$ & $\begin{array}{c}3.25 \pm \\
2.93\end{array}$ & $\begin{array}{c}24.25 \pm \\
14.16\end{array}$ \\
\hline P-value & 0.087 & 0.530 & 0.114 & 0.099 & 0.412 & 0.207 & 0.500 & 0.380 & 0.321 & 0.157 & 0.253 \\
\hline \multicolumn{12}{|c|}{ Level of education } \\
\hline Primary & $\begin{array}{c}12.20 \pm \\
8.48 \\
\end{array}$ & $3.82 \pm 3.23$ & $1.45 \pm 1.00$ & $6.94 \pm 5.43$ & $\begin{array}{c}14.31 \pm \\
13.27 \\
\end{array}$ & $3.55 \pm 4.11$ & $\begin{array}{c}10.76 \pm \\
9.58 \\
\end{array}$ & $\begin{array}{c}4.27 \pm \\
3.71 \\
\end{array}$ & $\begin{array}{c}3.31 \pm \\
3.68 \\
\end{array}$ & $\begin{array}{c}5.69 \pm \\
4.76 \\
\end{array}$ & $\begin{array}{c}39.78 \pm \\
31.11 \\
\end{array}$ \\
\hline Preparatory & $\begin{array}{c}10.42 \pm \\
6.69\end{array}$ & $3.95 \pm 3.28$ & $1.19 \pm 1.31$ & $5.28 \pm 3.73$ & $\begin{array}{c}13.72 \pm \\
10.40\end{array}$ & $4.21 \pm 3.99$ & $9.51 \pm 7.29$ & $\begin{array}{c}3.40 \pm \\
2.29\end{array}$ & $\begin{array}{c}1.09 \pm \\
2.04\end{array}$ & $\begin{array}{c}5.40 \pm \\
4.06\end{array}$ & \begin{tabular}{|c|}
$34.02 \pm$ \\
21.91 \\
\end{tabular} \\
\hline Secondary & $\begin{array}{c}15.08 \pm \\
13.79\end{array}$ & $5.62 \pm 5.81$ & $2.62 \pm 1.26$ & $6.85 \pm 7.16$ & $\begin{array}{c}15.92 \pm \\
19.02\end{array}$ & $4.92 \pm 7.16$ & $\begin{array}{c}11.00 \pm \\
11.94\end{array}$ & $\begin{array}{c}3.46 \pm \\
4.01 \\
\end{array}$ & $\begin{array}{c}3.08 \pm \\
4.68\end{array}$ & $\begin{array}{c}5.31 \pm \\
6.47 \\
\end{array}$ & $\begin{array}{c}42.85 \pm \\
46.93 \\
\end{array}$ \\
\hline Technical & $\begin{array}{c}17.53 \pm \\
9.11 \\
\end{array}$ & $5.87 \pm 3.93$ & $2.13 \pm 1.46$ & $9.53 \pm 4.58$ & $\begin{array}{c}22.80 \pm \\
14.09\end{array}$ & $8.67 \pm 5.35$ & $\begin{array}{c}14.13 \pm \\
8.90\end{array}$ & $\begin{array}{c}4.20 \pm \\
3.28 \\
\end{array}$ & $\begin{array}{c}4.07 \pm \\
3.24 \\
\end{array}$ & $\begin{array}{c}7.33 \pm \\
4.56 \\
\end{array}$ & $\begin{array}{c}55.93 \pm \\
32.57 \\
\end{array}$ \\
\hline P-value & 0.073 & 0.282 & $0.004 *$ & 0.052 & 0.090 & 0.002* & 0.393 & 0.752 & $0.002 *$ & 0.389 & 0.103 \\
\hline
\end{tabular}

Mann-Whitney test (for age and sex)

* Statistical significant difference $(P<0.05)$

N.B: The higher score the more problems

Table (5): Relationship of demographic data and domains of Multidimensional Child and Adolescent Depression Scale (MCADS) at Assiut City (N=120).

\begin{tabular}{|c|c|c|c|c|c|c|c|c|c|}
\hline \multirow[t]{2}{*}{ Variables } & Pessimism & $\begin{array}{c}\text { Weak } \\
\text { concentration }\end{array}$ & $\begin{array}{c}\text { Sleep } \\
\text { Problems }\end{array}$ & Anhedonia & Fatigue & Loneliness & $\begin{array}{c}\text { Low } \\
\text { self- } \\
\text { esteem }\end{array}$ & $\begin{array}{c}\text { Somatic } \\
\text { complaints }\end{array}$ & $\begin{array}{c}\text { total } \\
\text { Depression } \\
\text { Score }\end{array}$ \\
\hline & $\begin{array}{c}\text { Mean } \pm \\
\text { SD }\end{array}$ & Mean \pm SD & $\begin{array}{c}\text { Mean } \pm \\
\text { SD }\end{array}$ & $\begin{array}{c}\text { Mean } \pm \\
\text { SD }\end{array}$ & $\begin{array}{c}\text { Mean } \\
\pm \text { SD }\end{array}$ & $\begin{array}{c}\text { Mean } \pm \\
\text { SD }\end{array}$ & $\begin{array}{l}\text { Mean } \\
\pm \text { SD }\end{array}$ & $\begin{array}{c}\text { Mean } \pm \\
\text { SD }\end{array}$ & $\begin{array}{c}\text { Mean } \pm \\
\text { SD }\end{array}$ \\
\hline \multicolumn{10}{|l|}{ Age: (years) } \\
\hline $\begin{array}{l}7-12 \\
\text { years }\end{array}$ & $\begin{array}{c}8.04 \pm \\
2.31\end{array}$ & $9.10 \pm 2.29$ & $\begin{array}{c}8.33 \pm \\
2.36\end{array}$ & $8.38 \pm 2.86$ & $\begin{array}{c}8.19 \pm \\
2.53\end{array}$ & $\begin{array}{c}7.85 \pm \\
2.69\end{array}$ & $\begin{array}{c}7.38 \pm \\
2.05\end{array}$ & $7.33 \pm 2.44$ & $\begin{array}{c}64.60 \pm \\
14.37\end{array}$ \\
\hline $\begin{array}{l}>12-17 \\
\text { years }\end{array}$ & $\begin{array}{c}9.01 \pm \\
2.28\end{array}$ & $9.58 \pm 2.39$ & $\begin{array}{c}8.67 \pm \\
2.28\end{array}$ & $9.86 \pm 2.45$ & $\begin{array}{c}9.43 \pm \\
2.13\end{array}$ & $\begin{array}{c}8.75 \pm \\
2.47\end{array}$ & $\begin{array}{c}8.31 \pm \\
2.13\end{array}$ & $8.65 \pm 2.73$ & $\begin{array}{c}72.26 \pm \\
13.22\end{array}$ \\
\hline P-value & 0.029* & 0.307 & 0.382 & $\mathbf{0 . 0 0 3}^{*}$ & $0.003 *$ & $0.037 *$ & 0.018* & 0.007* & 0.005* \\
\hline \multicolumn{10}{|l|}{ Sex: } \\
\hline Male & $\begin{array}{c}8.64 \pm \\
2.26\end{array}$ & $9.59 \pm 2.29$ & $\begin{array}{c}8.50 \pm \\
2.28\end{array}$ & $9.38 \pm 2.41$ & $\begin{array}{c}8.97 \pm \\
2.00\end{array}$ & $\begin{array}{c}8.76 \pm \\
2.58\end{array}$ & $\begin{array}{c}8.15 \pm \\
2.10\end{array}$ & $8.29 \pm 2.56$ & $\begin{array}{c}70.29 \pm \\
12.50\end{array}$ \\
\hline Female & $\begin{array}{c}8.60 \pm \\
2.49\end{array}$ & $9.02 \pm 2.44$ & $\begin{array}{c}8.60 \pm \\
2.38\end{array}$ & $9.05 \pm 3.22$ & $\begin{array}{c}8.86 \pm \\
2.96 \\
\end{array}$ & $\begin{array}{c}7.71 \pm \\
2.48 \\
\end{array}$ & $\begin{array}{c}7.52 \pm \\
2.18\end{array}$ & $7.81 \pm 2.92$ & $\begin{array}{c}67.17 \pm \\
16.74 \\
\end{array}$ \\
\hline P-value & 0.826 & 0.150 & 0.824 & 0.394 & 0.575 & 0.028* & 0.135 & 0.195 & 0.223 \\
\hline \multicolumn{10}{|l|}{ Place: } \\
\hline $\begin{array}{l}\text { Lillian } \\
\text { Trasher }\end{array}$ & $\begin{array}{c}9.02 \pm \\
2.29\end{array}$ & $9.53 \pm 2.41$ & $\begin{array}{c}8.65 \pm \\
2.26\end{array}$ & $9.32 \pm 2.59$ & $\begin{array}{c}9.14 \pm \\
2.30\end{array}$ & $\begin{array}{c}8.74 \pm \\
2.60\end{array}$ & $\begin{array}{c}8.14 \pm \\
2.24\end{array}$ & $8.26 \pm 2.60$ & $\begin{array}{c}70.80 \pm \\
14.01\end{array}$ \\
\hline Al-Hanan & $7.27 \pm$ & $9.80 \pm 2.01$ & $8.53 \pm$ & $10.20 \pm$ & $8.73 \pm$ & $7.93 \pm$ & $7.80 \pm$ & $8.93 \pm 3.26$ & $69.20 \pm$ \\
\hline
\end{tabular}




\begin{tabular}{|c|c|c|c|c|c|c|c|c|c|}
\hline & 1.87 & & 2.50 & 2.68 & 1.87 & 2.31 & 1.15 & & 11.07 \\
\hline Al-Safa & $\begin{array}{c}7.25 \pm \\
2.09\end{array}$ & $7.83 \pm 1.80$ & $\begin{array}{c}7.67 \pm \\
2.39 \\
\end{array}$ & $7.67 \pm 3.23$ & $\begin{array}{c}7.58 \pm \\
3.09\end{array}$ & $\begin{array}{c}6.25 \pm \\
1.66\end{array}$ & $\begin{array}{c}6.50 \pm \\
1.83 \\
\end{array}$ & $6.08 \pm 1.56$ & $\begin{array}{c}56.83 \pm \\
13.31\end{array}$ \\
\hline P-value & $0.002 *$ & 0.041* & 0.380 & 0.073 & 0.129 & 0.004* & 0.048* & 0.013* & 0.009* \\
\hline \multicolumn{10}{|c|}{ Level of education } \\
\hline Primary & $\begin{array}{c}8.12 \pm \\
2.37\end{array}$ & $8.92 \pm 2.23$ & $\begin{array}{c}8.33 \pm \\
2.42\end{array}$ & $8.45 \pm 2.85$ & $\begin{array}{c}8.24 \pm \\
2.54\end{array}$ & $\begin{array}{c}7.76 \pm \\
2.66\end{array}$ & $\begin{array}{c}7.33 \pm \\
2.02\end{array}$ & $7.41 \pm 2.47$ & $\begin{array}{c}64.55 \pm \\
14.45\end{array}$ \\
\hline Preparatory & $\begin{array}{c}8.91 \pm \\
2.15\end{array}$ & $9.37 \pm 2.30$ & $\begin{array}{c}8.70 \pm \\
2.22\end{array}$ & $9.56 \pm 2.31$ & $\begin{array}{c}9.12 \pm \\
2.21\end{array}$ & $\begin{array}{c}8.81 \pm \\
2.75\end{array}$ & $\begin{array}{c}8.28 \pm \\
2.12\end{array}$ & $8.30 \pm 2.69$ & $\begin{array}{c}71.05 \pm \\
13.55\end{array}$ \\
\hline Secondary & $\begin{array}{c}10.00 \pm \\
2.27\end{array}$ & $10.15 \pm 2.85$ & $\begin{array}{c}9.54 \pm \\
1.56 \\
\end{array}$ & $\begin{array}{c}11.00 \pm \\
2.77\end{array}$ & $\begin{array}{c}10.62 \pm \\
2.06\end{array}$ & $\begin{array}{c}9.31 \pm \\
1.93 \\
\end{array}$ & $\begin{array}{c}8.77 \pm \\
2.74 \\
\end{array}$ & $9.38 \pm 2.40$ & $\begin{array}{c}78.77 \pm \\
11.90 \\
\end{array}$ \\
\hline Technical & $\begin{array}{c}8.27 \pm \\
2.37\end{array}$ & $10.33 \pm 2.23$ & $\begin{array}{c}7.87 \pm \\
2.56\end{array}$ & $9.60 \pm 2.56$ & $\begin{array}{c}9.20 \pm \\
1.70\end{array}$ & $\begin{array}{c}8.47 \pm \\
2.00\end{array}$ & $\begin{array}{c}8.20 \pm \\
1.61\end{array}$ & $8.87 \pm 3.16$ & $\begin{array}{c}70.80 \pm \\
11.75\end{array}$ \\
\hline P-value & 0.060 & 0.133 & 0.127 & 0.020* & 0.010* & 0.076 & 0.066 & 0.049* & $0.007 *$ \\
\hline
\end{tabular}

Mann-Whitney test (for age and sex)

* Statistical significant difference $(P<0.05)$

Table (1): Showed that the demographic data of the studied orphans. The result reveals that three fifth of studied orphans $(60 \%)$ in the age group from $(>12$ 17 years old).Also $65 \%$ of studied orphans were males. Regarding level of education $40.8 \%$ of them were primary level education and $77.5 \%$ of the studied orphans from dar Lillian trasher.

Table (2): Showed descriptive statistics of psychological aspects the studied orphans. According to competence scale ,the lower score the more problem and it was found that the lowest mean score \pm SD was related to activities level $(6.06 \pm 4.06)$.On the other hand, the problems scale the higher score the more problem and it was found that the highest mean score \pm SD was related to aggressive behavior followed by anxiety/depression. The mean scores \pm SD of them $10.76 \pm 9.02 \& 6.66 \pm 5.12$ respectively.

Figure (1): demonstrated that the distribution of the studied orphans regarding child behavior checklist, it was founded that, more than one third $(37.5 \%)$ of them suffered from internalizing behavioral problems, while $(34.2 \%)$ of them suffered from externalizing behavioral problems and more than one third $(36.7 \%)$ were in abnormal range of total problem scale. On the other hand, $(15.8 \%)$ were in the abnormal range of the total competence scale.

Table (3): Showed that relation between demographic data and domains of total competence scale .There were no statistical significant differences between age and all domains of total competence scale except activities level $(\mathrm{p}=0.001 *)$. However there were statistically significant differences between sex and all domains of total competence scale except activities level. As regard to place, there were statistically significant difference with school performance and total competence scale $\left(\mathrm{p}=0.000^{*}\right)$, $\left(\mathrm{p}=0.014^{*}\right)$ respectively. While level of education was statistically and significantly related to all domains of total competence scale except social relationship.
Table (4): Showed that relation between demographic data and domains of total problems scale. According to child age, there were no statistically significant differences with all domains of total problems scale except delinquent behavior $\left(\mathrm{p}=0.008^{*}\right)$. While there were statistically significant differences between child sex and all domains of total problems scale. In relation to place, it was not that it was not related to all domains of total problems scale. While level of education, was not related to all domains of total problems scale except somatic complaints behavior, delinquent behavior and thought problems $(\mathrm{p}==0.004 *),(\mathrm{p}=0.002 *)$ and $(\mathrm{p}=0.002 *)$ respectively.

Table (5): Showed the relationship between demographic data and different domains of Multidimensional Child and Adolescent Depression Scale (MCADS). According to the child age, there was a statistically significant difference between child age and domains of (MCADS) except weak concentration and sleep problems. While there were no statistically significant differences between child sex and all domains of (MCADS) except loneliness $(\mathrm{p}=0.028 *)$.Regarding to place was statistically and significantly related to all domains of (MCADS) except sleep problems, anhedonia and fatigue. As regard to level of education, there were no statistically significant differences with all domains of (MCADS) except anhedonia, fatigue, somatic complaints and total depression Score $\left(\mathrm{p}=0.020^{*}\right)$, $\left(\mathrm{p}=0.010^{*}\right),\left(\mathrm{p}=0.049^{*}\right)$ and $(\mathrm{p}=0.007 *)$ respectively.

\section{Discussion}

There is a general agreement that parental deprivation has a traumatic experience on all stages in children's life, from newborn to adolescence. As well as those children have a high incidence of developmental delay and growth problems, behavioral, psychological, emotional and cognitive problems (Van Ijzendoorn et al., 2011). 
The current study was aimed to assess psychological aspects among children and adolescents of orphanages at Assiut City.

In the present study the most of studied orphans (two third) age group was from (>12-17 years old) compared with more than one third were from (7-12 year).These findings are similar with previous study reported by (Yendork \& Somhlaba, 2015), who found that the age for all orphans ranged from (7-17 years). (41\%) participants were aged between (7-12 years), Children whereas $(59 \%)$ were aged between (13-17years) adolescents.

The current study revealed that about two third of studied orphans were males compared with slightly more than one third were females. These findings are similar with previous study reported by (Yendork\& Somhlaba, 2015) who found that two third of orphans were males while females were more than one third. Also (Afework, 2013) found that the majority of studied orphans were males. In contrast (Saboula et al., 2015) who revealed that more than half of studied orphans were females. This may be attributed to cultures difference.

As regarding to place, the current study showed that three quarters of the studied orphans were from Lillian trasher. This may be related to that Lillian trasher is consider a big institution than El-Hanan and El-Safa dar and care with much numbers of orphans than any other place.

According to level of education, the current study showed that more than two fifths of studied orphans were primary education. This result is agreement with (Yendork \& Somhlaba, 2015) who found that most of orphan children were primary education. In the current study, the highest mean score \pm SD of psychological aspects was related to aggression, followed by anxiety/depression.10.76 $\pm 9.02 \& 6.66 \pm$ 5.12 respectively. This may be attributed to many factors as lack of communication skills between orphans and their caregivers, lack of love, security and orphan's view point that to be strong, should be aggressive. Also, one of the common mental health problems is depression that is found among orphanage-placed children (Hermenau et al., 2011).

The findings of the present study showed that slight less than two fifth of the studied sample suffered from internalizing behavioral problems which include withdrawn, somatic complaints and anxiety / depression, while more than one third of them suffered from externalizing behavioral problems which include delinquent and aggressive behavior. The internalizing problems were higher than externalizing ones (37.5\% versus $34.2 \%)$.This finding could be due to that overcrowding, inadequate personal attention and poor relationship between orphans and their caregivers and thus externalizing problems observed more than internalized problems. These findings are in agreement with (Lehmann et al., 2013) who reported that orphans are exposed to numerous emotional and behavioral difficulties such as anxiety, depression, aggression, and anti-social behavior. Also (Mutiso et al., 2017) who found that prevalence of internalizing problems was (36.8\%).

The present study showed that there were statistically significant differences between age groups and activity level as age group between 7-12 year had less activity than age group $>12-17$ year. This mean decrease in activities level among children than adolescents (The lower score the higher problems). These findings may attributed to that children in this age are fearful from punishment for small and simple matters and don't do any activity without asking their caregivers and this fear may prevent them from participate in activities with other. In addition, most institutions have rules and regulations that have to be followed by every child, thus providing the children with minimal opportunities for breaking rules (Mutiso et al., 2017). In the present study there were statistically significant difference between sex and domain of total competence scale that includes social relationship and school performance and mean \pm SD among males is less than in females which indicate higher problems among males than females. This may be attributed to many factors as number of most studied orphans were males. In additional to, view of points of orphans about view of society toward them "orphanage nurturing" stigma make them conflict with other and absenteeism from school especially males which affected on their social relationship and school performance.

In the current study, there were statistically significant differences between place and school performance. Those who lived in El-Hanan dar have the lowest mean, which mean higher problems were among them. During data collection researcher observed that, most of El-Hanan dar orphans are unconcerned about school and hopeless about anything, especially due to view of society regard them " orphanage nurturing " stigma which affect on their performance at school.

According to level of education, there was statistically significant difference between level of education and school performance, it was found that technical education orphans had the lowest mean which indicate the highest problem among them. This finding may be interpreted by that they may seek to earn money quickly whatever methods to not be controlled by their caregivers and they consider 
school is wasting time, it is not benefit as previous, also for whom they will success.

In the current study, the mean of the most predominant problem was related to aggression, anxiety/depression and attention problem whereas thought problem was the least, and males were higher in all domains. These findings agree with (Mutiso et al., 2017) who found boys had higher prevalence rates in all of the condition.

In the current study, there was statistically significant difference between age and delinquent behavior and orphan adolescents whose age is $>12-17$ year have delinquent behaviors higher than orphan children who age 7-12 year. This could be due to the effect of adolescence stage which is characterized by a period of exploration and experimentation with a variety of roles and behaviors as youth attempt to define their identity, delinquency is seen as manner to communicate for what they are feeling (Berger, 2009) These findings agree with (Doku, 2012) who found that there were statistically significant difference between age and delinquent behavior.

In the present study there was statistically significant relation between sex and all domains of total problem scale, and mean of males was higher in all domains of total problem scale than females. This finding may be interpreted by that males' viewpoint regarding their future that they will leave orphanage after certain age and no one will be responsible about them and this consider a big problem according to them, in contrast to females who are still in orphanage until their marriage, there will be other who will be responsible about them. These findings are in line with previous study done by (Yendork \& Somhlaba, 2015) who found that gender was significantly related with the total psychological distress. Also, (Strunk, 2006) found that prevalence of psychiatric problems is significantly higher in boys.

Also, there was statistically significant relation between level of education and somatic complaints behavior, delinquent behavior and thought problems. This finding may attributed to that physical health problems, negative thought and emotion can undermine orphan's ability to success in school and to maintain close relationship with other.

As regarding to Multidimensional Child and Adolescent Depression Scale, The current study reported that age was statistically significant differences with total depression score, pessimism, anhedonia, fatigue, loneliness and low self-esteem. Depression is significantly higher in adolescents than children; it may be due to their ability to express feelings than children.

This finding is nearly consistent with(Kazam \& Al zubaidi, 2011) who found that, age groups were statistically significant differences with total score, pessimism, anhedonia, and fatigue, with older children scoring higher in every case .

According to sex, the current study found that there was a statistical significant difference with loneliness and loneliness in males higher than females. This may be attributed to many factors as, high number of males in comparison to females. Also, it may be due to poor relationship with the staff, caregivers, lack of love from community or memory of parental loss (Sebsibe et al., 2014).

The current study reported that levels of education were statistically and significantly different with total depression score, and the highest mean was among secondary education. It may be related to that secondary level adolescents are stressed than all students especially orphans who lost family support. This finding is in agreement with study of (Kazim $\boldsymbol{\&}$ Mohamed, 2016) who found that significant relation in depression with educational level.

\section{Conclusion}

Based on the result of the present study we concluded that, most orphans had different psychological problems and the prevalence of psychological problems/symptoms was more in adolescence age than child ages.

\section{Recommendations}

- Psychosocial counseling program can be designed and administered to orphan children and adolescents to improve depressive symptom and their psychological status.

- Psycho- educational program can be prepared to those who are responsible about the orphanage to increase their knowledge toward the psychological problems of orphans.

- Orphanage must be provided with the recreational and social facilities that help the children to ventilate their energy in a healthy ways as (drawing, music, sports and needlework).

- Liaison psychiatric nurses should be available in orphanages to assess the psychological status of orphans and help them cope effectively.

- Further studies must be done to determine other psychological problems by other tools and in other Egyptian governorates.

\section{References}

1. Abdel-Khalek, A., (2003): The Multidimensional Child and Adolescent depression Scale: psychometric properties. Psychological Reports, 93,544-560.

2. Abdel-Khalek, E., (2006): Magnitude of Violence, Its Determinants and Impact on Health among Secondary School Students in Assiut 
Governorate. Thesis Doctorate Degree in Public health and Community Medicine, Faculty of Medicine, Assiut University, p.p1-13.

3. Achenbach, T., \& Rescorla, L., (2001): Child Behavior Checklist (CBC) for ages (6-18), University of Vermont, ed.201, pp.1-4.

4. Akram, M., Anjum, F., \& Akram, N., (2015): Role of Orphanages to Uplift the SocioEconomic Status of Orphans Focusing on SOS Children's Villages in Punjab, Pakistan. Mediterranean Journal of Social Sciences, 6(3 S2), 177.

5. Berens, A., \& Nelson, C., (2015): The science of early adversity: is there a role for large institutions in the care of vulnerable children? The Lancet, 386(9991), 388-398.

6. Berger, K., (2009): The developing person through childhood and adolescence. (8 ed.). New York: Worth Pub.

7. Berk, L., (2002): Infants and children: Prenatal through middle childhood (4th ed.). Boston: Allyn \& Bacon.

8. Birmaher, B., \& Brent, D., (2010): Depression and dysthymia. Dulcan's Textbook of Child and Adolescent Psychiatry, 261-265.

9. Blaikie, P., Cannon, T., Davis, I., \& Wisner, B., (2014): At risk: natural hazards, people's vulnerability and disasters. Routledge.

10. Boadu, O., (2015): A Comparative Study of Behavioral and Emotional Problems among Children Living in Orphanages in Ghana: A Mixed Method Approach (Doctoral dissertation, University of Ghana).

11. Boris, G., (2012): Post Orphanage Behavior Syndrome. Retrieved from /www.bgcentrt.com/BG Publications/Orphanage behavior.html.

12. Boris, N., Brown, L., Thurman, T., Rice, J., Snider, L. M., Ntaganira, J., \& Nyirazinyoye, L., (2008): Depressive symptoms in youth heads of household in Rwanda -Correlates and implications for intervention. Archives of Pediatrics \& Adolescent Medicine, 162(9), 836843.

13. Cluver, L., Gardner, F., \& Operario, D., (2007): Psychological distress amongst AIDS-orphaned children in urban South Africa. Journal of child psychology and psychiatry, 48(8), 755-763.

14. Coghlan, A., (2014): A psychotherapeutic exploration of the long-term effects of the death of a parent in childhood.

15. De Witt, M., \& Lessing, A., (2010): The psychosocial well-being of orphans in Southern Africa: the perception of orphans and teachers. TD: The Journal for Trans disciplinary Research in Southern Africa, 6(2), 461-477.

16. Doku P., (2012): The Mental Health of Orphans and Vulnerable Children within the context of HIV/AIDS in Ghana, doctor thesis.

17. Hermenau, K., Hecker, T., Ruf, M., Schauer, E., Elbert, T., \& Schuaer, M., (2011): Childhood adversity, mental ill-health and aggressive behaviour in an African orphanage: Changes in response to trauma-focused therapy and the implementation of a new instructional system. Child and Adolescent Psychiatry and Mental Health, 5, 29. doi:10.1186/1753-2000-529.

18. Hopkin, J., (2008): Emergency Mental Health and Psychological Support., Public Health Guide in Emergency (2nd ed):198-219.

19. Jameel, S., Shah, S. A. \& Ganaie, S. A. (2015). Perceived Social Support and Resilience among Orphans: A Systematic Review.

20. Kazam, A., \& Al Zubaidi, A., (2011): Depression symptoms among Omani Children: Age and Sex Differences. Psychological Report:.Doi:10.2466/02.09.17.PR0.108.3.805812.source: PubMed.

21. Kazim, A., \& Mohamed, S., (2016): Assessment of Psychological Status of Orphans in Orphanages in Baghdad City, kufa journal for nursing sciences vol.6 No. 1.

22. Kostopoulos, A., (2014): Getting Their Stories: Narratives of Youths in a Shelter for Orphaned and Vulnerable Children. M.A. Thesis. South Africa: University of Witwatersrand.

23. Lehmann, S., Havik, O., Havik, T., \& Heiervang, E., (2013): Mental disorders in foster children: A study of prevalence, comorbidity and risk factors. Child and Adolescent Psychiatry.

24. Louw, D., \& Louw, A., (2007): Child and adolescence development. Bloemfontein: Psychology Publications.

25. Luchterhand, C., \& Murphy, N., (2013): Helping adults with mental retardation grieve a death loss. Taylor \& Francis.

26. Melhem, N., Porta, G., Shamseddeen, W., Payne, M., \& Brent, D.,. (2011): Grief in children and adolescents bereaved by sudden parental death. Archives of general psychiatry, 68(9), 911-919.

27. Mohammadzadeh, M., Awang, H., Shahar, H., \& Ismail, S., (2017): Emotional health and selfesteem among adolescents in Malaysian orphanages. Community mental health journal, 19

28. Mohammed, K., (2001): Psychiatric Aspect of Violence among Preparatory School Students; Thesis Doctorate Degree in Psychiatry, Faculty of 
Medicine, Assiut University, p.p1-5.

29. Mohammed, S., Mosallem, F., Mohammed, N., \& Mohammed, A., (2013): "Assessment of Social Support and Psychological Distress among Adolescents in Foster Care at El-Minia Governorate" master thesis.

30. Mutiso, V., Musyimi, C., Tele, A., \& Ndetei, D., (2017): Epidemiological patterns and correlates of mental disorders among orphans and vulnerable children under institutional care, Soc Psychiatry Psychiatric Epidemiol 52:65-75.

31. Sebsibe, T., Fekadu, D., \& Molalign, B., (2014): Psychosocial wellbeing of orphan and vulnerable children at orphanages in Gondar Town, North West Ethiopia. Journal of Public Health and Epidemiology, 6(10), 293301. http://doi.org/10.5897/JPHE 2014.0648.

32. Shaik Omar, N., (2016): Fostering roles and adopting identities: exploring the effect of drama therapy on the sense of identity of Muslim orphaned and vulnerable adolescents (Doctoral dissertation).

33. Strunk, D., Lopez, H., \& DeRubeis, R., (2006): "Depressive symptoms are associated with unrealistic negative predictions of future life events". Behavior Research and Therapy, Vol.44, No.11, P.896.

34. Van Ijzendoorn, M., Palacios, J., Sonuga-Barke, E., Gunnar, M., Vorria, P., McCall, R., \& Juffer, F., (2011): I. Children in institutional care: Delayed development and resilience. Monographs of the Society for Research in Child Development, 76(4), 8-30.

35. Yendork, J., \& Somhlaba, N., (2015): The Role of Demographic and Positive Psychological Factors on Quality of Life in Ghanaian Orphans Placed in Orphanages: An Exploratory Study., Child Ind Res (2016) 9:429-443. 